\title{
Mitochondrial transplantation: Another miracle of molecular medicine?
}

\author{
S. Ram Kumar, MD, PhD, FACS
}

From the Division of Cardiac Surgery, Department of Surgery, University of Southern California, Heart Institute, Children's Hospital of Los Angeles, Los Angeles, Calif.

Disclosures: Author has nothing to disclose with regard to commercial support.

Received for publication March 23, 2017; accepted for publication March 24, 2017; available ahead of print April $27,2017$.

Address for reprints: S. Ram Kumar, MD, PhD, FACS, Children's Hospital, Los Angeles, 4650 Sunset Blvd, Mailstop \#66, Los Angeles, CA 90027 (E-mail: rsubramanyan@ chla.usc.edu).

J Thorac Cardiovasc Surg 2017;154:284-5

$0022-5223 / \$ 36.00$

Copyright (c) 2017 by The American Association for Thoracic Surgery

http://dx.doi.org/10.1016/j.jtcvs.2017.03.074

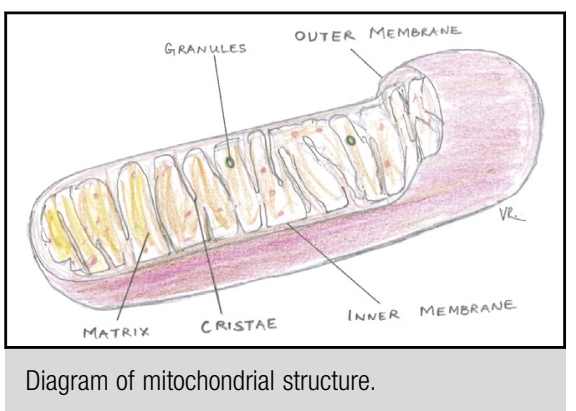

Among myriad evolutionary alterations, an adult human heart, unlike its counterpart in zebrafish or newt, lost its ability to effectively regenerate and replenish lost or dysfunctional cardiomyocytes. Heart muscle damage, and consequent heart failure, therefore continues to be a vexing clinical problem with limited traditional therapeutic options. Medical management relies on drugs that aim to augment the performance of the remaining functional cardiomyocytes. Surgical management replaces the entire organ to provide a new, functioning unit. Over the past few years, rapid insights gained into molecular biology in general, and cardiac molecular physiology in particular, have paved the way for a number of novel biological therapies to address heart failure. Probably the most widely known among them is cell-based regenerative therapy. Multiple strategies, ranging from providing a sheet of functional mature cardiomyocytes to implanting exogenous progenitor or stem cells capable of forming new cardiomyocytes, have been attempted and some early success has been demonstrated in a few clinical trials. Despite improvement in heart function, these exogenous cells have not been reliably shown to differentiate into cardiomyocytes and integrate into the host heart. Therefore, alternative approaches directed at stimulating proliferation of progenitor cells residing within the heart, or inducing other cell types such as fibroblasts to differentiate into functional cardiomyocytes, have also been pursued. Preclinical studies that edit the diseased gene in the heart, or replace genes or gene products that can improve heart muscle regeneration, have all been undertaken with guarded success. Less well explored is the idea of replenishing subcellular organelle fractions that can provide the most crucial function to limit the effect of major injury.

Based on well-designed preclinical evidence, ${ }^{1}$ Emani and colleagues $^{2}$ have ventured to do exactly that in a pilot clinical trial. In the setting of acute myocardial ischemia (diagrammatically represented in Figure 1), studies have shown that there is injury to mitochondria associated with a rapid decline in high-energy phosphates that provide fuel to cardiomyocytes. In a rabbit model of cardiac ischemia-reperfusion, Masuzawa and colleagues ${ }^{1}$ injected autologous mitochondria isolated from pectoralis muscle into the myocardium just before reperfusion. This resulted in improved myocardial recovery without any adverse event. Emani and colleagues ${ }^{2}$ report the use of this technique in 5 children who required extracorporeal support secondary to heart failure purportedly resulting from ischemia that was surgically addressed. There was no demonstrable short-term adverse effect and 4 of these patients showed evidence of improvement in heart function and could be weaned from mechanical support. This work is clearly preliminary and suffers from important limitations. First, there was a small number of patients, and no control arm. There were no uniform criteria to ensure ischemia-reperfusion in all patients. Most importantly, a causative link between mitochondrial transplantation and subsequent clinical improvement cannot be made. In its current form, this study should best be viewed as a feasibility first-in-human protocol. Much needs to be done before this can be accepted as a therapeutic tool. The fundamental question not answered in this study is the mechanism by which mitochondrial injection improves heart function. The authors speculate on the possibilities by extrapolating from animal data. However, there are some fundamental differences. In their animal study, the injections were undertaken before reperfusion, whereas in these children, injections were undertaken 2 to 15 days after surgically addressing myocardial ischemia. The immediate effect of supplanting high-energy substrates and precursor 


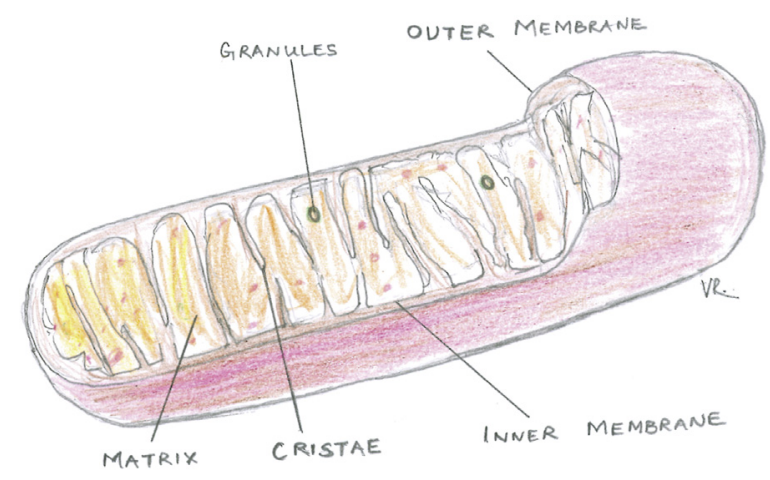

FIGURE 1. Diagram of mitochondrial structure.

metabolites are likely less relevant in the human study because cellular damage from ischemia-reperfusion probably was complete before mitochondria were injected. The animal studies showed that $<10 \%$ of injected mitochondria were eventually internalized by the cardiomyocytes. Whether such a small number of internalized mitochondria is sufficient to integrate and favorably improve cardiomyocyte metabolism and function remains to be elucidated.

Regardless of its limitations, this innovative study reaffirms our faith that the next wave of novel therapeutic agents is coming in the form of biologic therapy. Molecular medicine continues to hold the promise of providing new approaches to tackle challenging clinical conditions. The authors should be commended for pushing the boundaries and elegantly demonstrating that it is up to us to embrace this transformation and adhere to responsible innovation. It is time for us to mature into translational therapists; that is, practitioners who can translate discoveries at the bench into cures at the bedside.

\section{References}

1. Masuzawa A, Black KM, Pacak CA, Ericsson M, Barnett RJ, Drumm C, et al Transplantation of autologously derived mitochondria protects the heart from ischemia-reperfusion injury. Am J Physiol Heart Circ Physiol. 2013;304:H966-82. 2. Emani SM, Piekarski BL, Harrild D, del Nido PD, McCully JD. Autologous Mitochondrial transplantation for dysfunction following ischemia reperfusion injury. J Thorac Cardiovasc Surg. 2017;154:286-9. 